\title{
Impacto de la intervención de enfermería en el autocuidado de pacientes con enfermedad renal crónica avanzada
}

\author{
Luis Huaman-Carhuas ${ }^{1,2}$, Hugo F. Gutiérrez-Crespo ${ }^{3}$ \\ ${ }^{1}$ Facultad de Enfermería. Universidad Peruana Cayetano Heredia. Lima. Perú \\ ${ }^{2}$ Hospital Nacional Alberto Sabogal Sologuren. Callao. Perú \\ ${ }^{3}$ Facultad de Medicina de la Universidad Nacional Mayor de San Marcos. Lima. Perú
}

\begin{abstract}
Como citar este artículo:
Huaman-Carhuas L, Gutiérrez-Crespo HF. Impacto de la intervención de enfermería en el autocuidado de pacientes con enfermedad renal crónica avanzada.

Enferm Nefrol. 2021 Ene-Mar;24(1):68-76
\end{abstract}

\section{Resumen}

Introducción: Los pacientes con enfermedad renal crónica avanzada ven gravemente afectada su calidad de vida lo que a menudo puede implicar riesgo de muerte.

Objetivo: Evaluar el impacto de la intervención de enfermería en el autocuidado de pacientes con enfermedad renal crónica avanzada de un hospital público.

Material y Método: Estudio pre experimental de corte longitudinal y prospectivo. La muestra incluyó a 60 pacientes en estadíos 3b, 4 y 5 de la enfermedad renal crónica, a quiénes se aplicó 3 tipos de cuestionario previamente validados, antes y después de la intervención de enfermería que consistió en tres sesiones educativas y asesoramiento en consulta externa. Las tres dimensiones que se tomaron para la investigación fueron: conocimiento sobre la enfermedad, autocuidados y adherencia farmacológica. Para el análisis de datos, en conocimiento se utilizó la prueba de Wilcoxon; en autocuidados, la prueba T de Student, y la prueba de McNemar para medir la adherencia. Para el procesamiento de resultados se utilizó el software SPSS v.20.

\section{Correspondencia:}

Luis Huaman Carhuas

Email: luis.huaman.c@upch.pe
Resultados: Tras la intervención, el conocimiento sobre autocuidado se incrementó hasta un $71,7 \%$, con relación al momento pre-intervención. Igualmente, el nivel de autocuidado aumentó tras la intervención a $72 \%$. Respecto a los adherentes al tratamiento farmacológico, antes de la intervención eran el 5\%, posteriormente se incrementó hasta el $65 \%$.

Conclusión: La intervención de enfermería basada en actividades educativas y de asesoramiento produce un impacto positivo en el autocuidado de pacientes con enfermedad renal crónica avanzada.

PALABRAS CLAVE: autocuidado; enfermedad renal crónica; consulta de enfermería; estrategia de salud.

Impact of the nursing intervention on the selfcare of patients with advanced chronic kidney disease

\section{Abstract}

Introduction: Patients with advanced chronic kidney disease have a severely affected quality of life, which can often imply the risk of death.

Objective: To evaluate the impact of the nursing intervention in the self-care of patients with advanced chronic kidney disease in a public hospital. 
Material and Methods: Pre-experimental longitudinal and prospective study. The sample included $60 \mathrm{pa}-$ tients with stages $3 b, 4$ and 5 of chronic kidney disease. Three types of previously validated questionnaires were applied before and after the nursing intervention, which consisted of three educational sessions and outpatient counseling. The three dimensions that were taken for the research were: knowledge about the disease, self-care and pharmacological adherence. For data analysis, the Wilcoxon test was used in knowledge; in self-care, the Student's $t$ test, and the Mcnemar test to measure adherence. For the processing of results, the SPSS v.20 software was used.ce. SPSS v.20 software was used to process the results.

Results: After the intervention, knowledge about self-care increased to $71.7 \%$, in relation to the pre-intervention moment. Likewise, the level of self-care increased after the intervention to $72 \%$. Regarding those people who showed adherence to pharmacological treatment, before the intervention they were $5 \%$, and later the percentage increased to $65 \%$.

Conclusion: The nursing intervention based on educational and counseling activities produces a positive impact on the self-care of patients with advanced chronic kidney disease.

KEYWORDS: self-care; chronic kidney disease; nursing consultation; health strategy.

\section{Introducción}

La Enfermedad Renal Crónica (ERC) viene generando el aumento de pacientes de manera exponencial en todo el mundo ${ }^{1}$, convirtiéndose en un serio problema de salud pública debido a las elevadas tasas de incidencia, morbimortalidad y sobrecarga de los sistemas de salud². La ERC se clasifica, según el filtrado glomerular en cinco etapas o estadíos. Estas etapas están asociadas con un mayor riesgo de morbilidad cardiovascular, mortalidad prematura y / o disminución de la calidad de vida, debido a que la enfermedad suele progresar de manera asintomática hasta los estadíos avanzados ${ }^{3}$ donde adquiere la condición de Enfermedad Renal Crónica Avanzada (ERCA).

La ERC tiene una alta prevalencia global estimada entre el 11 y el $13 \%$, con la mayoría en estadío 3, siendo amplia la variabilidad de la prevalencia si se toma en cuenta factores geográficos, género y económicos, des- de $8,8 \%$ en algunos países hasta $12,5 \%$ en otros 3,4 . A nivel de Perú, no existen estudios de prevalencia de la ERC en sus diferentes estadíos; solo existen tres estudios que muestran tasas referenciales, siendo el último del año 2011 dónde se obtuvo cifras porcentuales de 2 ciudades. En base a los datos de la Encuesta NHANES de los EEUU, aunque con ciertas limitaciones, se estima en promedio que un 2.5 millones de personas tendrían ERC en estadíos avanzados ${ }^{5}$.

Según algunos estudios, los pacientes con ERCA experimentan la aparición de complicaciones clínicas y alteraciones analíticas que condicionan a un ingreso no programado en terapias de reemplazo renal ${ }^{6,7}$. Esta situación muy frecuente, que afecta la calidad de vida de los pacientes con ERCA, se ve influenciada por el aumento de la esperanza de vida y las comorbilidades asociadas como la diabetes, hipertensión arterial y enfermedades cardiovasculares ${ }^{8,9}$.

Las diferentes manifestaciones clínicas de la ERCA comienzan paralelamente a la progresión del estadío; a partir de los estadíos 4 y 5, cuando el filtrado glomerular ha descendido por debajo de $30 \mathrm{ml} / \mathrm{min}$, aparecen la mayoría de los síntomas presentes en la enfermedad renal, incrementándose su severidad a medida que avanza la nefropatía. A nivel sistémico podemos observar alteraciones en el sistema nervioso, en el sistema hematológico e inmunológico, en el sistema cardiovascular, en el sistema digestivo y en el sistema endocrino ${ }^{10}$.

Las situaciones antes descritas ofrecen a enfermería una oportunidad de intervenir a través de la consulta de ERCA en las áreas de prevención, elección, inducción de la terapia, y otros autocuidados, utilizando para ello principalmente el componente educativo, y actuando en equipo multidisciplinar ${ }^{11}$. El autocuidado, fundamentado por Dorothea Orem, es una función humana reguladora que debe aplicar cada individuo de forma deliberada con el fin de mantener su vida y su estado de salud, desarrollo y bienestar, por tanto, es un sistema de acción. Como función reguladora del hombre, el autocuidado es diferente de otros tipos de regulación del funcionamiento y el desarrollo humano ${ }^{12}$. Esta teoría ofrece a los profesionales de la enfermería herramientas para una atención de calidad, en cualquier situación relacionada con el binomio salud-enfermedad ${ }^{13}$.

En los pacientes con ERCA los temas abordados en el autocuidado incluyen el cumplimiento de las medidas preventivas de nefroprotección, la práctica de actividad física, alimentación adecuada, abandono del taba- 
co y alcohol, control de la presión arterial y su registro permanente entre otros. Estos cambios necesarios se tornan más difíciles de mantener en el tiempo, especialmente si el paciente no es consciente de la importancia que tienen en su vida, además de no contar con las herramientas necesarias para abordar las acciones de cuidado. Además de esto, existe evidencia de que las personas manifiestan el deseo de mejorar la resolución de problemas para alcanzar los objetivos propuestos para su salud, ${ }^{14}$ por lo que los programas educativos impartidos a través de las consultas son importantes y pueden beneficiar a todos aquellos pacientes susceptibles de este tipo de intervención que debe efectuarse de forma continua y sin grandes intervalos de tiempo ${ }^{15}$.

A nivel local, es muy frecuente el ingreso de pacientes con ERCA estadío 5, a los hospitales para requerir hemodiálisis de urgencia mediante catéter venoso central no tunelizado, con una carga de complicaciones y descompensación clínica asociada a comorbilidades severas, escaso conocimiento y medidas de autocuidado, sin manejo especializado previo en consulta prediálisis, lo que incide directamente en la supervivencia y calidad de vida ${ }^{7}$. Debido a la importancia de la ERCA y su repercusión en el estilo y calidad de vida de los enfermos renales, es imprescindible conocer en nuestro medio, si la actividad 0 enseñanza de enfermería en consulta ERCA ofrece beneficios a los pacientes. Por tanto, el objetivo del presente estudio fue evaluar el impacto de la intervención de enfermería en el autocuidado, basada en actividades educativas y de asesoramiento, de pacientes con enfermedad renal crónica avanzada, de un hospital público.

\section{Material y Método}

\section{Diseño, ámbito y duración del estudio}

Estudio de nivel aplicativo, diseño pre experimental de un solo grupo, corte longitudinal y prospectivo en una población de pacientes con ERCA del hospital Alberto Sabogal Sologuren de la provincia Callao - Perú, Ilevado a cabo durante el año 2018.

\section{Población}

La población de estudio estuvo comprendida por los pacientes con ERCA estadíos 3b, 4 y 5 que acudieron a la consulta ERCA y cumplieron con los criterios de selección.

\section{Criterios de selección}

Los criterios de inclusión fueron: pacientes con diagnóstico de ERC estadíos 3b, 4 y 5, acreditados al hos- pital de estudio y que aceptaron participar voluntariamente con la firma de consentimiento informado. Dentro de los criterios de exclusión estuvieron los que padecían algún tipo de neoplasia avanzada, afección severa de las capacidades visual, auditiva y física. El período de enrolamiento y duración del estudio fue 1 año.

\section{Variables de estudio e Instrumentos de medida \\ - Variable Independiente, intervención de enfermería educativa.}

- Variable dependiente. Autocuidado de pacientes con ERCA prediálisis, donde se incluyeron las dimensiones de conocimiento sobre la enfermedad, práctica del autocuidado y adherencia farmacológica. Dentro del componente conocimiento, se indagó sobre la enfermedad renal crónica, factores relacionados, complicaciones y prevención; en cuanto a la práctica de autocuidados se recogieron datos sobre actividad física, hábitos de alimentación, hábitos nocivos, control de peso y presión arterial.

La intervención de enfermería se aplicó siguiendo una guía de procedimientos institucional y un módulo educativo del seguro social, que consistió en 3 sesiones educativas y de asesoramiento. Durante el tiempo de estudio, se desarrolló la captación, intervención y seguimiento de cada paciente hasta completar el estudio. En una etapa previa a la intervención, se tomó el pre test y después de la última sesión se aplicó el post test.

Para la recolección de datos se utilizaron 3 instrumentos. El instrumento referido a conocimientos y practica de autocuidados elaborado por los autores (anexo), fue validado previamente mediante juicio de expertos y una prueba piloto antes de su aplicación definitiva. Con el fin de determinar la confiabilidad del instrumento, para la variable conocimiento se aplicó la prueba estadística Kuder y Richardson fórmula 20, obteniéndose un resultado de 0,71 considerado alto; y para la variable práctica de autocuidados se aplicó Alpha de Crombach obteniéndose $œ=0,709$, por lo que se concluye que el instrumento es aceptable para medir los objetivos de la investigación. Además, se utilizó el Cuestionario de adherencia Morisky-Green-Levine ${ }^{18}$ para evaluar el cumplimiento farmacológico de los pacientes en estudio, test indirecto validado y usado en numerosos estudios que consta de cuatro preguntas de respuesta dicotómica sí o no sobre sus actitudes ante la medicación de forma entremezclada durante la entrevista clínica. 


\section{Métodos estadísticos}

Para el comparativo entre pre y post intervención de enfermería, variable conocimiento, se empleó la prueba no paramétrica de Wilcoxon de los rangos con signo; para la variable autocuidado, se utilizó la prueba paramétrica $\mathrm{T}$ de medidas relacionadas y para la tercera variable adherencia terapéutica se empleó la Prueba de McNemar.

\section{Aspectos/consideraciones éticas}

De acuerdo con las normas internacionales de investigación la información obtenida fue manejada de forma estrictamente confidencial, salvaguardando en todo momento el anonimato del paciente en estudio. El estudio se coordinó con la oficina de capacitación y ética del hospital en estudio. La participación de los pacientes fue previa firma del consentimiento informado con libre derecho de elegir y retirarse del estudio.

\section{Resultados}

Del total de pacientes participantes en el estudio que fueron 60 en todos los estadíos, el $75 \%$ tienen edades entre 60 y 80 años, un $15 \%$ son mayores de 80 años y el $10 \%$ pacientes con edades menores a 60 años. Los pacientes, según estadíos de la ERCA con mayor proporción se encuentran en el estadío $3 b$ seguido del estadío 4 y estadío 5 como se muestra en la Figura 1.

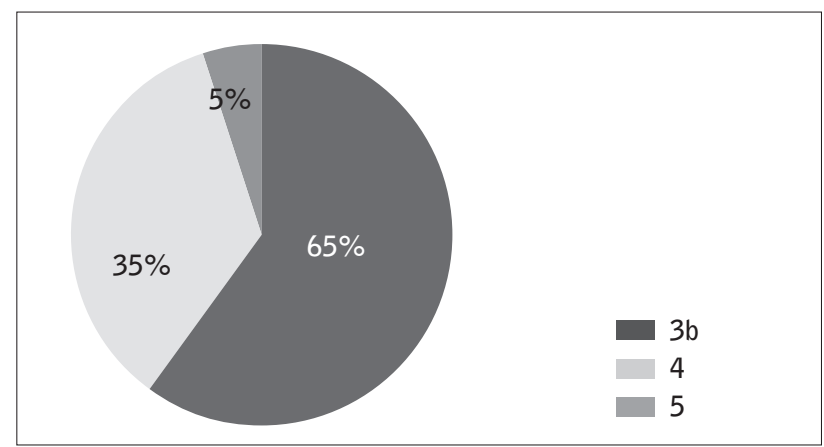

Figura 1. Distribución de pacientes con ERCA según estadíos.
Respecto al nivel de conocimiento sobre la enfermedad, se evidenció que antes de la intervención no se encontraron pacientes con conocimiento alto, predominando en mayor proporción pacientes con conocimiento bajo. Después de la intervención se observó que el nivel de conocimiento bajo disminuyó y el nivel de conocimiento alto se incrementó a 71,7\% (Tabla 1). Utilizando la prueba Wilcoxon se estableció que existe una diferencia estadística entre ambos momentos de estudio $(p<0,001)$.

En cuanto a la práctica de autocuidados, en la fase pre intervención hubo predominio de un nivel bajo y medio, ambos representaron a la totalidad de pacientes del estudio. Tras la intervención se evidenció el aumento del autocuidado alto y se redujo el autocuidado bajo, en tanto el autocuidado medio se mantuvo con ligera reducción como se observa en la Tabla 2.

El resultado del estudio en cuanto a la adherencia terapéutica evidenció que al inicio la mayor parte de pacientes con ERCA tuvieron condición de no adherentes, y en el periodo post intervención los pacientes con adherencia a la terapéutica se incrementaron mientras que los no adherentes disminuyeron (Tabla 3).

\section{Discusión}

El objetivo del estudio fue determinar el impacto de la intervención de enfermería en los pacientes con ERCA, observándose un mayor porcentaje de pacientes en el estadio $3 b$ respecto a los estadíos 4 y 5 , ratificando la presencia de una lesión renal de rápido avance en esta fase de la enfermedad. Por ello, es importante realizar una adecuada valoración a los pacientes en este estadío, debido a la aparición de múltiples complicaciones y desarrollar intervenciones con el objetivo de enlentecer la progresión de la enfermedad renal. Diversas investigaciones han reportado los beneficios de la intervención de enfermería en estos pacientes tratados en base a la

Tabla 1. Nivel de conocimiento antes y después de la intervención de enfermería.

\begin{tabular}{|c|c|c|c|c|c|}
\hline \multirow[t]{2}{*}{ Nivel de conocimiento } & \multicolumn{2}{|c|}{ Antes de intervención } & \multicolumn{2}{|c|}{ Después de intervención } & \multirow{2}{*}{$\begin{array}{c}\text { Estadístico } \\
\mathbf{p}\end{array}$} \\
\hline & Frecuencia & Porcentaje & Frecuencia & Porcentaje & \\
\hline Conocimiento bajo & 51 & $85,0 \%$ & 1 & $1,7 \%$ & \\
\hline Conocimiento regular & 9 & $15,0 \%$ & 16 & $26,6 \%$ & $p<0,001$ \\
\hline Conocimiento alto & 0 & $0,0 \%$ & 43 & $71,7 \%$ & \\
\hline Total & 60 & $100 \%$ & 60 & $100 \%$ & \\
\hline
\end{tabular}

a: Prueba de Wilcoxon de los rangos con signo, nivel de significancia $œ=0,05$. Conocimiento total post - Conocimiento total pre (Sig. Asintótica bilateral). 
Tabla 2. Nivel de autocuidado antes y después de la intervención.

\begin{tabular}{|c|c|c|c|c|c|}
\hline \multirow[t]{2}{*}{ Nivel de autocuidado } & \multicolumn{2}{|c|}{ Antes de intervención } & \multicolumn{2}{|c|}{ Después de intervención } & \multirow{2}{*}{$\begin{array}{c}\text { Estadístico } \\
\mathbf{p}\end{array}$} \\
\hline & Frecuencia & Porcentaje & Frecuencia & Porcentaje & \\
\hline Autocuidado bajo & 39 & $65,0 \%$ & 2 & $3 \%$ & \\
\hline Autocuidado medio & 21 & $35,0 \%$ & 15 & $25 \%$ & $<0,001$ \\
\hline Autocuidado alto & 0 & $0,0 \%$ & 43 & $72 \%$ & \\
\hline Total & 60 & $100 \%$ & 60 & $100 \%$ & \\
\hline
\end{tabular}

a: Prueba paramétrica T, nivel de significancia $œ=0,05$. Autocuidado total post - Autocuidado total pre. (sig. Bilateral).

Tabla 3. Adherencia terapéutica antes y después de la intervención.

\begin{tabular}{|c|c|c|c|c|c|}
\hline \multirow[t]{2}{*}{ Adherencia } & \multicolumn{2}{|c|}{ Antes de intervención } & \multicolumn{2}{|c|}{ Después de intervención } & \multirow{2}{*}{$\begin{array}{c}\text { Estadístico }^{\mathrm{a}} \\
\mathbf{p}\end{array}$} \\
\hline & Frecuencia & Porcentaje & Frecuencia & Porcentaje & \\
\hline No adherente & 57 & $95,0 \%$ & 21 & $35 \%$ & \multirow{2}{*}{$<0,001$} \\
\hline Adherente & 3 & $5 \%$ & 39 & $65 \%$ & \\
\hline Total & 60 & $100 \%$ & 60 & $100 \%$ & \\
\hline
\end{tabular}

a: Prueba de McNemar, nivel de significancia $œ=0,05$. Adherencia pre \& Adherencia post.

enseñanza en consulta externa, ya que les permite planificar con antelación la creación del acceso vascular, minimizar las complicaciones, retardar la progresión de la enfermedad y controlar mejor los parámetros de laboratorio ${ }^{18}$. Sin embargo, existe un porcentaje muy bajo de pacientes que son atendidos en la consulta ERCA por parte de enfermería, esto es debido a que, esta consulta al día de hoy no está implantada de forma sistemática en todos los centros de referencia nefrológicos ${ }^{19}$. Un estudio chileno sobre intervención de enfermería, en la ERCA a través de la consulta, demostró ser eficaz para retrasar la progresión de la ERC en adultos que están en la fase prediálisis, por lo que la consulta liderada por el profesional de enfermería es extremadamente útil, ya que dedica el tiempo necesario para dar seguimiento a las intervenciones educativas, cerciorándose que entienda y asimile los conocimientos para una mejor adherencia a los cuidados $^{11}$. Los resultados presentan coincidencias con reportes descritos por Cases-Amenós et al. ${ }^{20}$ donde un $61,5 \%$ de los pacientes presentaron ERC en estadío 3, un 30,2\% se hallaban en estadío 4 y un $8,3 \%$ restante en estadío 5 .

La intervención de enfermería sobre el nivel de conocimientos del paciente ha producido cambios significativos, pues la prueba basal pre intervención evidenció que el $85 \%$ de los pacientes estudiados evidenciaron tener conocimiento bajo sobre cómo caracterizar la enfermedad renal crónica, reconocimiento de signos y síntomas, complicaciones, tratamiento y medidas de autocuidado, lo cual refleja que la situación de pacientes adscritos al Sistema de Seguridad Social, tiene escasa exposición a información preventiva sobre enfermedades crónica degenerativas, o por lo contrario, supone escasa oferta y estrategia por parte de las entidades de salud para impulsar la salud renal. Cuando se analizan los resultados tras la enseñanza, comprobamos que el conocimiento alto sube hasta $71,7 \%$, lo que indica la influencia de la intervención. Estos datos fueron similares con otros hallazgos de estudios sobre intervención educativa de enfermería en el conocimiento sobre ERC, donde se presente incrementos hasta el $90 \%$ respecto a la fase pre intervención ${ }^{21-23}$.

En relación a la práctica de autocuidado, la intervención educativa de enfermería desarrollada, logró un incremento en las puntaciones de autocuidado, lo cual ratifica la existencia de un cambio positivo en la conducta de los pacientes respecto al autocuidado. La implementación de programas educativos a través de la consulta ERCA, incide de manera importante en las actividades básicas del cuidado como alimentación, cumplimiento terapéutico y ejercicios ${ }^{15,24,25}$. Es necesario enfatizar el rol de educador que los profesionales de enfermería realizan dentro del contexto predialítico, no sólo para el control y prevención de complicaciones propias de la enfermedad, sino también, como un sistema de apoyo del componente subjetivo del paciente para mejorar su bienestar y calidad de vida, 
ya que las intervenciones educativas constituyen una importante herramienta a tomar en cuenta en el control de patologías crónica degenerativas desde los primeros niveles de atención ${ }^{26,23}$.

La falta de adherencia al tratamiento farmacológico sigue siendo un problema prevalente en la práctica clínica, especialmente en el tratamiento de enfermedades crónicas. En la consulta ERCA es posible identificar el cumplimiento terapéutico y detectar las dificultades del paciente, lo cual puede evitar o minimizar los fracasos terapéuticos, reducir la estancia hospitalaria y costes sanitarios, con ello se contribuye a mejorar la calidad de vida de los pacientes ${ }^{27}$. El resultado de este estudio respecto a la toma de medicamentos, especialmente nefro protectores para controlar niveles de tensión arterial, glicemia, lípidos y otros según la prescripción médica, llamó la atención el escaso número de pacientes con adherencia en el pre test si tenemos en cuenta que muchos de ellos tuvieron consulta médica previa. Tras la intervención de enfermería, se evidenció una mejoría en la adherencia al tratamiento que supera el $60 \%$, resultados que evidenciaron cambios en la conducta del grupo de pacientes en estudio respecto al cumplimiento de la prescripción de los medicamentos, lo cual se asemeja a lo encontrado en el estudio de 0jeda y Pérez ${ }^{15,28}$.

A partir de los resultados podemos sostener que la intervención de enfermería basada en actividades educativas y de asesoramiento desarrolladas en la consulta ERCA, produce impacto positivo en el autocuidado de pacientes mejorando el conocimiento sobre la enfermedad y la adherencia al tratamiento farmacológico.

Recepción: 11-10-20

Aceptación: 10-01-21

Publicación: 30-03-21

\section{Bibliografía}

1. Ayar Y, Ersoy A, Ocakoglu G, Yildiz A, Oruc A, Soyak $\mathrm{H}$ et al. Risk Factors Affecting Graft and Patient Survivals After Transplantation From Deceased Donors in a Developing Country: A Single-Center Experience. Transplant Proc. Elsevier USA; 2017 Mar $1 ; 49(2): 270-77$.

2. Cruz Vera FES, Tagliamento G, Wanderbroocke AC. A manutenção da vida laboral por doentes renais crônicos em tratamento de hemodiálise: Uma análise dos significados do trabalho. Saude e Soc. Univ Sao Paulo; 2016 0ct 1;25(4):1050-63.

3. Hill NR, Fatoba $S T$, Oke JL, Hirst JA, $0^{\prime}$ Callaghan CA, Lasserson DS, Hobbs FDR. Global prevalence of chronic kidney disease - A systematic review and meta-analysis. PLoS ONE. Public Library of Science; 2016.

4. Mills KT, Xu Y, Zhang W, Bundy JD, Chen CS, Kelly TN, Chen J, He J. A systematic analysis of worldwide population-based data on the global burden of chronic kidney disease in 2010. Kidney Int. Nature Publishing Group; 2015 Nov 1;88(5):950-57.

5. Loza Munariz C, Ramos Muñoz W. Análisis de la situación de la enfermedad renal crónica en el Perú, 2015 [Internet]. 2016 [Consultado 29 junio 2020]. Disponible en: https://www.spn.pe/archivos/ANALISIS\%20DE\%20LA\%20SITUACION\%20DE\%20 LA\%20ENFERMEDAD\%20RENAL\%20CRONICA\%20EN\%20\%20EL\%20PERU\%20(1).pdf.

6. Sarrias Lorenz X, Bardón Otero E, Vila Paz M. EI paciente en pred-diálisis: toma de decisiones y libre elección terapéutica. Nefrologia. 2008; Supl. 3:S119-23.

7. Huamán C L, Postigo O C, Contreras C C. Características epidemiológicas de los pacientes que inician hemodiálisis crónica en el Hospital Alberto Sabogal Sologuren 2015. Horiz méd. 2016;16(2):6-12.

8. Shafiee MA, Akbarian F, Memon KK, Aarabi $M$, Boroumand B. Dermatologic manifestations in end-stage renal disease. Iran $\mathrm{J}$ Kidney Dis. 2015;9(5):339-53.

9. Parrado MDCR, Pozo MG, Garrido MC, Tendero CT, Montero RC. Analysis of the quality of life of the patient in the predialysis stage. Enferm Nefrol. Sociedad Española de Enfermería Nefrológica; 2017;20(3):233-40. 
10. Galperin TA, Cronin AJ, Leslie KS. Cutaneous Manifestations of ESRD. Clin J Am Soc Nephrol. 2014 Jan 7;9(1):201-18.

11. Forero J, Barrios S. Rol de enfermería en la consulta de prediálisis en el paciente con enfermedad renal cónica avanzada. Enfermería Nefrológica. 2016;19(1):77.

12. Acosta MP. Explorando la teoría general de enfermería de Orem Exploring the general theory of nursing Orem. Ensayo Enf Neurol (Mex). 2011.

13. Naranjo Hernández Y, Concepción Pacheco J, Rodríguez Larreynaga M. The self-care deficit nursing theory: Dorothea Elizabeth Orem. Gac Médica Espirituana. 2017;19(3).

14. Santana MBA, Silva DMGV da, Echevarría-Guanilo ME, Lopes SGR, Romanoski PJ, Böell JEW. Self-care in individuals with chronic kidney disease on hemodialysis. Rev Gauch Enferm. NLM (Medline); 2020;41:e20190220.

15. Ojeda Ramírez MD, Caro Rodríguez I, Ojeda Ramírez D, García Pérez A, García Hita S, García Marcos S. Nursing consultation and therapeutic adherence of the hemodialysis patient. Enferm Nefrol. 2017;20(2):132-38.

16. Andreu Periz L, Sarria Guerrero J. Farmacoterapia en la Enfermedad Renal. Adherencia terapéutica. Enfermería Nefrológica. 2017;20(4):367-70.

17. Pagès-Puigdemont N, Valverde-Merino MI. Métodos para medir la adherencia terapeútica. Ars Pharm. 2018 Sep 14;59(3):163-72.

18. Torres Torradeflot C, Gutiérrez Vilaplana J, Craver Hospital L, Baigol Guilanyà M. Resultado de la intervención Enseñanza: Dieta prescrita en la consulta de enfermedad renal crónica avanzada. Enferm Nefrol. 2016 Jan;19(1):12-9.

19. Rebollo Rubio A, Morales Asensio JM, Pons Raventos ME. Influencia de la consulta de enfermería de enfermedad renal crónica avanzada en pacientes que inician tratamiento renal sustitutivo. $243 \mathrm{En}$ ferm Nefrol. Sociedad Española de Enfermería Nefrológica; 2014 0ct 1;17(4):243-50.

20. Cases-Amenós A, Martínez-Castelao A, Fort-Ros $J$, Bonal-Bastons J, Ruiz MP, Vallés-Prats $M$ et al. Prevalencia de anemia y su manejo clínico en la enfermedad renal crónica estadios 3-5 no en diálisis en cataluña: Estudio MICENAS I. Nefrologia. 2014;34(2):189-98.
21. Boté Fernández C. Intervención educativa sobre la erc en atn primaria. Rev Soc Esp Enferm Nefrol [Internet]. 2009;12(4):250-52. Disponible en: http://scielo.isciii.es/pdf/nefro/v12n4/carta2.pdf.

22. Góngora Gómez 0, Jesús W, Carralero R, Saavedra Muñoz LB, Milord RB, Gómez Vázquez E. Intervención educativa sobre insuficiencia renal crónica en pacientes con diabetes mellitus tipo 2. Univ Médica Pinareña. Universidad de Ciencias Médicas de Pinar del Rio; 2019;15(2):184-93.

23. Montes De Oca Rodríguez SM, Gómez RV. Intervención Educativa para el control de la hipertensión arterial. Arch del Hosp Univ "General Calixto García." 2017;4(3):7-14.

24. Moses M, Olenik NL. Perceived impact of caregiver's participation in diabetes education classes on implementation of self-care behaviors. J Am Pharm Assoc. Elsevier B.V.; 2019 Jul 1;59(4):S47-S51.el.

25. Puello Alcocer EC, Amador Ahumada C, Ortega Montes JE. Impacto de las acciones de un programa de enfermería con enfoque promocional y de autocuidado en la capacidad funcional de adultos mayores. Univ y Salud. Universidad de Narino; 2017;19(2):152-62.

26. Díaz Piñera A, Salvá AR, Roche RG, García IC, Estupiñán FA. Resultados de una intervención para la mejora del control de la hipertensión arterial en cuatro áreas de salud. Rev Finlay. 2018;8(3):180-9.

27. Raventós Torner R, Borruel Llovera A, Raigal Ara L, Borreguero Guerrero E, Casado Martínez M, Ferré Grau C. La adherencia al tratamiento farmacológico en las consultas enfermeras de atención primaria: observar y comprender [Rev Rol Enferm.2020]-Medes [Internet]. [Consultado 7 Sep 2020]. Disponible en: https://medes.com/publication/151974.

28. Pérez Rosabal E, Soler Sánchez $Y$, Hung Fonseca Y, Rondón Zamora M. Programa educativo para favorecer la adherencia terapéutica en pacientes con tratamiento antirretroviral. Rev Arch Médico Camagüey. 2016;20(2):177-87. 
ANEXO:

CUESTIONARIO SOBRE AUTOCUIDADOS EN PACIENTES CON ENFERMEDAD RENAL CRÓNICA AVANZADA (ERCA)

\section{CUESTIONARIO I}

I. CONOCIMIENTOS: En cada ítem marcar con ASPA (X) la respuesta que considere correcta:

1. ¿Cuál es la función principal del riñón?

Regular el peso del cuerpo

Formar las toxinas de los alimentos

Regular la presión arterial

Desconozco

2. ¿Qué entiende por Enfermedad Renal Crónica?

Cuando los riñones ya no funcionan, pero

puede curarse

Cuando los riñones funcionan normal, sólo

disminuye la cantidad de orina

Cuando los riñones van dejando de funcionar

y no tiene cura

Desconozco

3. Las causas más frecuentes de la Enfermedad Renal Crónica son:

Consumir comida chatarra

Golpes y accidentes

Diabetesypresiónalta

Desconozco

4. Los signos y síntomas de la Enfermedad Renal Crónica avanzada son:

Fiebre, dolor de espalda

Hinchazón de pies, cambios en la orina.

Tos, dolor de cabeza.

Desconozco

5. Cuando los riñones dejan de funcionar,

¿Qué alternativas de reemplazo conoce?

Tratamiento médico y rehabilitacion

Acupuntura y medicina tradicional

Diálisis y trasplante Renal.

Desconozco.

6. La frecuencia recomendada para hacer ejercicio físico debe ser:

Una vez al día

Una vezala semana

Unavezal mes

Cuando se pueda

7. El tiempo mínimo de actividad física recomendada diaria debe ser:

5 minutos

10 minutos

20 minutos

30 minutos.

8. Para mantener un peso saludable se necesita:

Hacer dieta y no comer carne roja

Tomar más agua y menos carbohidratos

Llevar una vida sedentaria y vegetariana

Alimentición saludable y hacer ejercicios

\section{El consumo recomendado de la sal en}

la comida diaria, debe ser:

Menos de 6 gramos

Entre 6 - 10 gramos

Entre 11 - 15 gramos

Desconozco

10. ¿Cuál de las siguientes considera Ud. comida rápida?:

Sopa, ensalada de fruta

Helados, gaseosa, pan

Ensalada de verduras y guiso de Menestras

Pizza, hamburguesa, pollo broaster

11. Para prevenir la aparición de cálculos renales,

se recomienda:

Consumir más vitaminas

Disminuir ingesta de verduras y frutas

Disminuir consumo de café

Disminuir consumo de carnes rojas y sus

12. El consumo frecuente de alcohol puede afectar a:

Corazón

Riñones

Pulmones

Páncreas

13. ¿Cuál de los siguientes factores agrava más

el daño renal?

No tomar agua suficiente.

Consumir o exponerse al tabaco

Realizarexcesivoejerciciofísico

Comer frecuentemente en la calle

14. La presión arterial normal en adultos es:

$80 / 50 \mathrm{mmHg}$

$120 / 80 \mathrm{mmHg}$

$130 / 90 \mathrm{mmHg}$

$140 / 90 \mathrm{mmHg}$

15. ¿Cuántas dosis de vacuna se requieren

para estar protegidos contra la hepatitis $\mathbf{B}$ ?:

Una dosis

Dos dosis

Tres dosis

Desconozco

16. Los medicamentos prescritos por el nefrólogo que está tomando actualmente sirven para:

Regenerar células del riñón .

Aumentar la cantidad de orina.

Recuperar la función renal

Proteger al riñón y retardar su avance. 


\section{PRÁCTICA DE AUTOCUIDADOS: Marque con ASPA (X) la respuesta que mejor se ajuste a su realidad:}

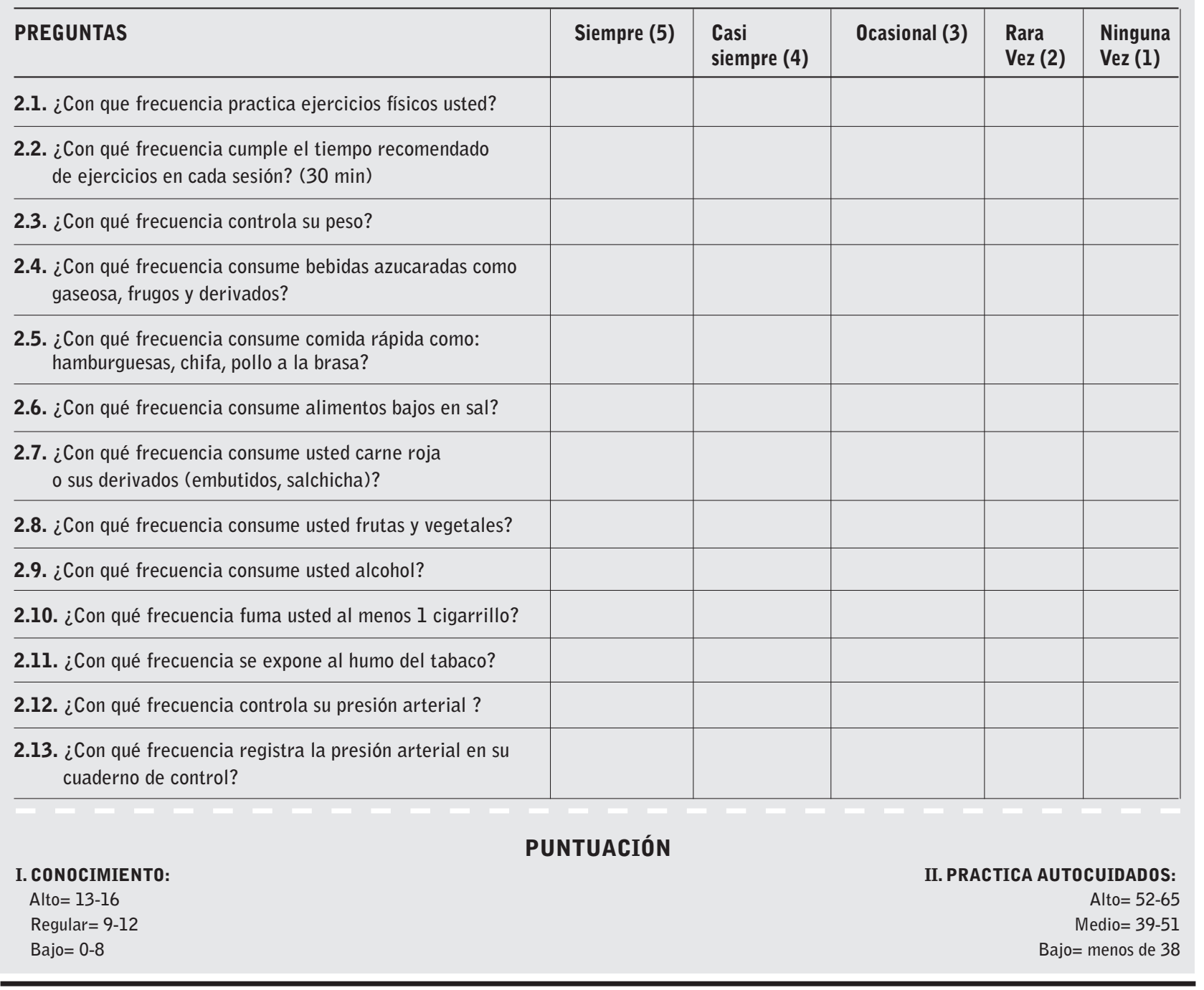

Este artículo se distribuye bajo una Licencia Creative Commons Atribución-NoComercial 4.0 Internacional. https://creativecommons.org/licenses/by-nc/4.0/

\section{Open Access (c) $\underset{\mathrm{EY}}{(\mathbf{\mathrm { NC }}}$}

